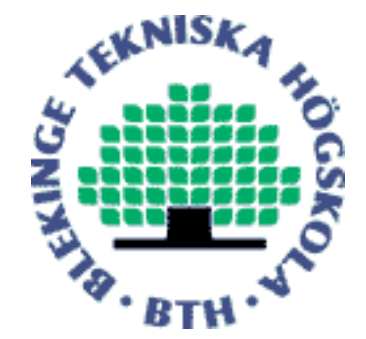

Copyright (C) 2010 IEEE.

Citation for the published paper:

On user perception of web login - a study on QoE in the context of security

Charlott Lorentzen, Markus Fiedler, Henric Johnson, Junaid Shaikh, Ivar Jørstad

ATNAC

2010 Auckland, NZ

This material is posted here with permission of the IEEE. Such permission of the IEEE does not in any way imply IEEE endorsement of any of BTH's products or services Internal or personal use of this material is permitted. However, permission to reprint/republish this material for advertising or promotional purposes or for creating new collective works for resale or redistribution must be obtained from the IEEE by sending a blank email message to pubs-permissions@iee.org.

By choosing to view this document, you agree to all provisions of the copyright laws protecting it. 


\title{
On User Perception of Web Login - A Study on QoE in the Context of Security
}

\author{
Charlott Lorentzen*, Markus Fiedler*, Henric Johnson*, Junaid Shaikh* and Ivar Jørstad ${ }^{\dagger}$ \\ ${ }^{*}$ School of Computing \\ Blekinge Institute of Technology \\ Karlskrona, Sweden \\ Email: firstname.surname@bth.se \\ †Ubisafe AS, Oslo, Norway \\ Email: ivar@ubisafe.no
}

\begin{abstract}
From the user point of view, security has shown to be ambivalent; on one hand it is felt necessary, but on the other hand it is felt disturbing. Authentication solutions, for instance, are designed to keep undesired and unauthorized users out; however, allowed users need to spend some effort and waiting time when logging into a system. The question remains to which extent this effort is perceived as positive as security is increased, or negative through the waiting time spent in the process. Excessive waiting times imply the risk of user churn.

As there is a lack of such studies, this paper investigates user perception (Quality of Experience, QoE) of the response times (Quality of Service, QoS) of a web authentication procedure, in particular a login to a community web page. Comparing the results to well-known user perception of web performance, we show that the users perceive logins in a similar way as standard web pages, which means that similar limits on user patience apply. The derived QoE-QoS relationship, an exponential function, serves then as the basis for assessing the performance of authentication algorithms in the domain of user acceptability.
\end{abstract}

\section{INTRODUCTION}

There are a few new authentication services coming up, of which OpenID [1] is one. This authentication is a solution that makes password management easy for the user. Only one username is required to $\log$ into several web pages once the user is authenticated to his/her OpenID provider. More of these security solutions are expected in the future, where ease of use is prioritized.

The demand of secure login schemes increases and the users' willingness to accept the security service depends on the ability to meet the users' expectations. For that reason, defining User Experience (UE) or Quality of Experience (QoE) [2] in combination with a security system is essential, both quantitative and qualitative. However, defining and measuring QoE is a challenge and involve interdisciplinary research since some of these measures are "soft" and not as concrete as researchers within e.g. telecommunication would like performance measures to be.

Quality of Service (QoS) has been a central research topic in telecommunication systems for a long time, and now the idea of QoS extended to QoE and UE is an emerging area.

User perception of web pages and perception of download times have also been evaluated and discussed as QoE in several studies such as [3], [4], and [5]. While user perception of regular web pages is pretty well known, perception of security enabled web pages, e.g. web pages requiring authentication, is hardly addressed in literature.

QoE of a service is usually evaluated based on the time it takes for the service to perform a given task, i.e. the response time (RT), which is the Quality of Delivery (QoD). QoS problems (such as loss, delay and other variations) leads to increased delivery time or response time (RT), i.e. QoD problems, which then translate into QoE. Thus, the QoE measure is the user's perception and judgement of the QoD, and the QoD is affected by QoS parameters.

In this paper $^{1}$, we evaluate QoE in the context of authentication. How do users experience the RT for security in the form of authentication? If a user log into a web page, for example a community like Facebook, how is the login time perceived in the context of feeling secure? Responses to and rankings of perceived security are searched for. A comparison is then performed to previous studies in QoE of web pages. Are the perceptions of security different from perception of other information technology experiences? And if so, to which extent?

From the answers to these questions and from the performed experiments, we aim to find a model for user perception of security in web pages. This model will be presented in this paper, together with usage possibilities and constraints. The user model will, in the proceeding of the work presented in this paper, be used to further evaluate similar authentication methods for Internet services.

The organization of this paper is as follows: Section II clarifies technical terms that are used throughout the paper. Section III describes the methodology of the study and Section IV describes the experiments with corresponding setup, procedure and RT measurements. The results (qualitative and quantitative) are presented in Section V, followed by a discussion of the results in Section VI. Finally, Section VII provides a conclusion of the paper and points out future work.

\footnotetext{
${ }^{1}$ This study is part of a larger study on evaluating authentication methods for an IMS platform [6], where QoE is one of the important criteria. The study was performed within the EUREKA!-funded Mobicome project.
} 


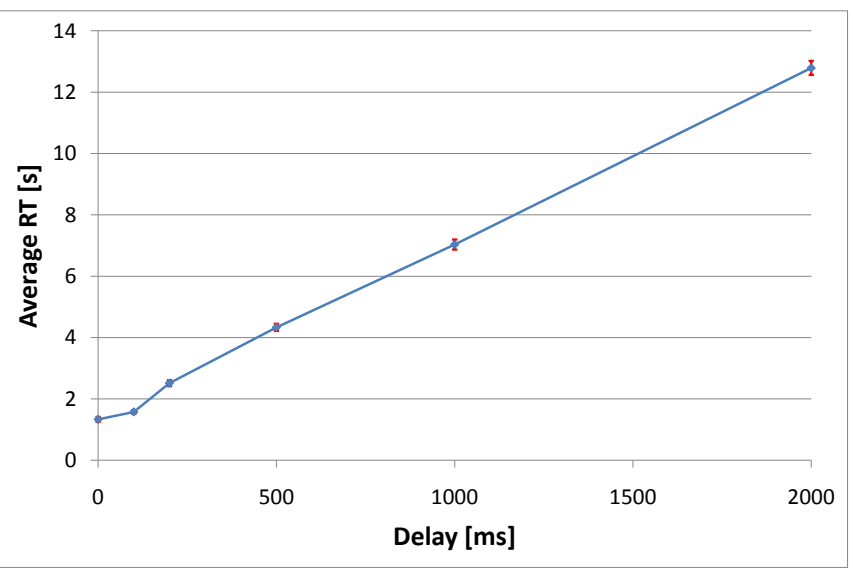

Fig. 1. Average RTs mapped to the corresponding added delay.

\section{TECHNICAL BACKGROUND}

\section{A. OpenID}

An OpenID identity is a unique URL which contains the trusted provider and the username. The provider is the host of the URL. In this case the provider is Ubisafe AS, in Norway, and the username/URL will be openid.ubisafe.no/ $<$ username $>$, but the provider can also be for example Yahoo, or any other site that provides OpenID as authentication service. With OpenID one can use the same password or authentication credential and username for every site that one has to authenticate oneself to.

If a user is going to use OpenID, e.g. for www.facebook.com, then he or she first has to enable OpenID at that web page. Then, in the future, when the user logs into openid.ubisafe.no (or the provider in question) it is possible to visit www.facebook.com and provide the OpenID username/URL, and the login to www.facebook.com will be completed without an additional password. This applies for all OpenID enabled pages during one web browsing session.

OpenID is an open standard for authentication and neither the protocol nor the web pages that support OpenID can put constraints on how to authenticate oneself. So the choice of authentication method is free.

\section{B. Quality of Experience}

The notion of QoE and UE have been widely discussed in [7], where the concept of QoE refers to the totality of end user experience of the delivered service [8].

Another definition of QoE is found in a recommendation [2] from ITU-T, where it is written "The overall acceptability of an application or service, as perceived subjectively by the enduser". As described in a recommendation [9] from ITU-T, the QoE can be measured by user tests and is then typically expressed with a Mean Opinion Score (MOS). There exist other methods to estimate QoE such as the E-model [10], instrumental metrics (PESQ) [9], and even a neural network approach [11], but these are mostly used for voice and video.

We denote, in this paper, the user perception and user experience by the term QoE. For evaluating the latter we use

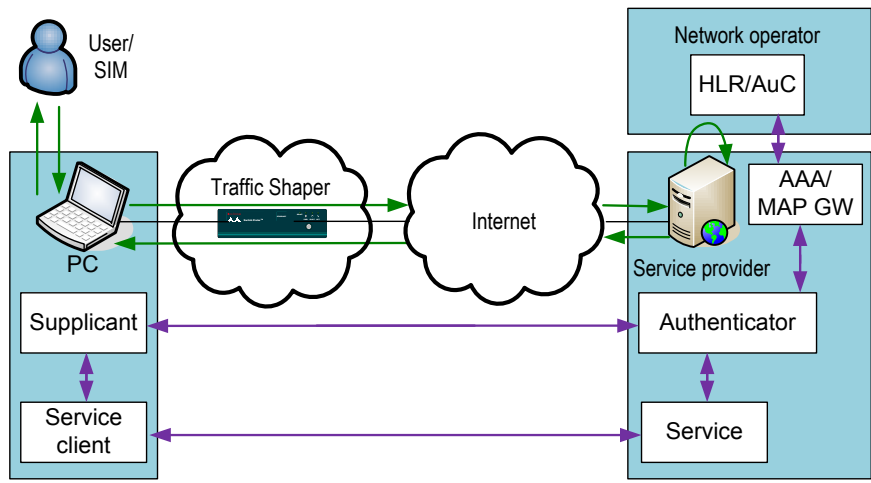

Fig. 2. Setup for confirm and login experiments.

TABLE I

INTERPRETATION OF MOS GRADES [12].

\begin{tabular}{|c||c|c|c|c|c|}
\hline MOS & 1 & 2 & 3 & 4 & 5 \\
\hline Interpretation & Bad & Poor & Fair & Good & Excellent \\
\hline
\end{tabular}

MOS, with the preset grades in a recommendation [12] from ITU-T, seen in Table I.

Several studies, by both academia and industry, have shown that users loose more interest the longer the RT of the service is. A user browsing a web page notices a couple of $100 \mathrm{~ms}$ of delay, gets bored after about 4 seconds, and after 10 seconds the user might leave the page [13].

\section{Service supply chains}

The total RT for a task in a service can consist of times from all parts of a Service Chain (SC) [14], which can for example be a client that send a request to a server, that in turn has to call another server or database to retrieve information to complete the request from the client. A SC can be very large and also quite complex.

In [14] it can be concluded that SCs are susceptible to growth in delays, and this can also be seen in Fig. 1, showing the average RT for the login procedure in this study. When adding a delay of $1 \mathrm{~s}$, the average RT is about $7 \mathrm{~s}$, and when adding a delay of $2 \mathrm{~s}$ the RT is about $13 \mathrm{~s}$. This SC mostly consists of a large chain of messages going to and from the SIM-dongle during the authentication procedure, while showing only one RT to the user since it is all contained in the same task. Though, as can be seen in Fig. 2, SCs for this service can also be quite complex for some tasks, with authentication requests going all the way to the HLR (Home Location Registry) at the network operator that issued the SIM card.

\section{Methodology}

This study uses measurements of RTs and user rankings of those, which is included in the methodology (see Fig. 3) of the larger study, to quantify user experience. The experiments aimed to give quantitative results on visiting security enabled web pages of the considered system, including the login procedure. 
A web login application will be used as a test case to find out the user satisfaction, the QoE, with regards to the RT. For a web login, the QoE is mainly affected by the delay of reply messages after a request has been submitted.

\section{A. Quantitative}

Quantitative results were retrieved from RT measurements on application level, during the experiments. For each experiment the time for starting and completing a task was logged. From these, a total time for completing each task could be calculated, which is what we call the RT of the system for that particular task. The RT for a particular task can be different each time the task is performed, since the total RT depends on, not only the authentication method, but on the network, the different servers, and also to some extent on the load on the client machine, i.e. on the whole SC for that task.

MOS is a mean value of the Opinion Scores (OS) given by users. It has been used in several studies before, amongst others to evaluate RT in a map service [4], and to evaluate RTs of web pages [5] and download times [3]. In our case the users rank their experience of a web page login procedure. Delay is put on the procedure in order to increase the RT. The user will then rank that experience according to the MOS-scale (see Table I), and the ranking will be mapped to the RT of that particular trail.

\section{B. Qualitative}

After the ranking, users were asked questions of more qualitative character of how they perceived the login procedure and the service, with regards to security. The questions were asked in order to find out the user perception of the service itself and also to underline the result of the user rankings.

\section{EXPERIMENTS}

Two sets of experiments were performed in the study. The performance of the system is shown in Fig. 1, were it can be seen that the actual RTs for the respective delays were larger than the added delay. Sometimes single RTs were as high as 16 seconds, which is retrieved because of the SC of messages needed in the authentication method. The first experiments considered a full OpenID login including the visited community web page before and after the user log into the OpenID server. The second experiment considered only the login procedure on the OpenID server web page, but the total procedure from the first experiment was still shown and explained to the user, to create an understanding of what they were testing.

\section{A. Setup of browsing experiment}

The first experiment setup consisted of a client computer with a SIM dongle, a traffic shaper for adding delay on the network interface of the client, and a server situated in Oslo, Norway, as shown in Fig. 2. All computers in the setup had a fixed line network connection.

The experiment setup in this study closely resembles a reallife setup, where server and client/user are situated on different

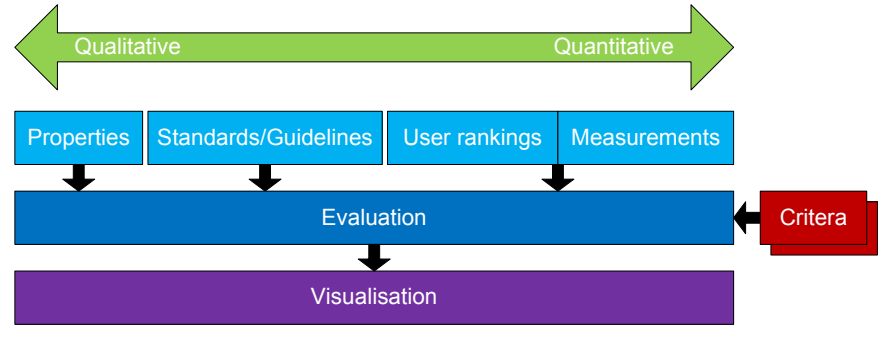

Fig. 3. Methodology for the study of authentication schemes [6].

geographical sites, as opposed to a setup in a regular laboratory environment where client and server are situated in the same room or in some cases even on the same computer. The only "artificial" part in this experiment setup is the traffic shaper, which is needed to provoke longer RTs. The delays that were added in the shaper were $0 \mathrm{~ms}, 100 \mathrm{~ms}, 200 \mathrm{~ms}, 300 \mathrm{~ms}, 500$ $\mathrm{ms}, 1 \mathrm{~s}$ and $2 \mathrm{~s}$.

In total there were 35 participants in the experiment, all students at Blekinge Institute of Technology, with varying experience of web services, and of ages between 22 and 30 years.

After telling the user about the procedure and OpenID, the user browsed to MyGeolog.com. There he or she chose OpenID as login method and entered the given user name. When clicking login, the user was then redirected to the web page of the OpenID server. Here "SIM-dongle" was chosen as authentication method and when clicking login, the server and the SIM dongle exchanged information in order to authorize the user via the SIM card, and give access to the original website. Then the user pressed confirm and he or she was redirected to the original website.

This total procedure is done only once. Then, when visiting the community again the user only needs to choose OpenID as login procedure at the community and then confirm their identity.

After performing each test (i.e. one login procedure) the user was asked to rank his/her experience. The tasks that were ranked are the confirm task together with the displaying of the MyGeolog.com page when the user is logged in. In other words, the last part of the authentication and the redirection, which is perceived as one task by the user, are ranked together. Even though the authentication has already been made at this stage, this is not obvious to the user since there is still the task of confirming one's identity before the page of MyGeolog.com is shown. I.e. this task is perceived as the last part of the authentication, or login.

A modified version of Fasterfox [15] was used in Firefox to get the RTs of the web pages. RTs were recorded and logged to a file on the client. Each RT was then mapped to the opinion score that the user provided for that particular test.

\section{B. Setup of login experiment}

The second experiment setup consisted of a client with a SIM dongle, a traffic shaper for setting the bandwidth (BW) for the client, thus inducing delay, and a server, still situated in 


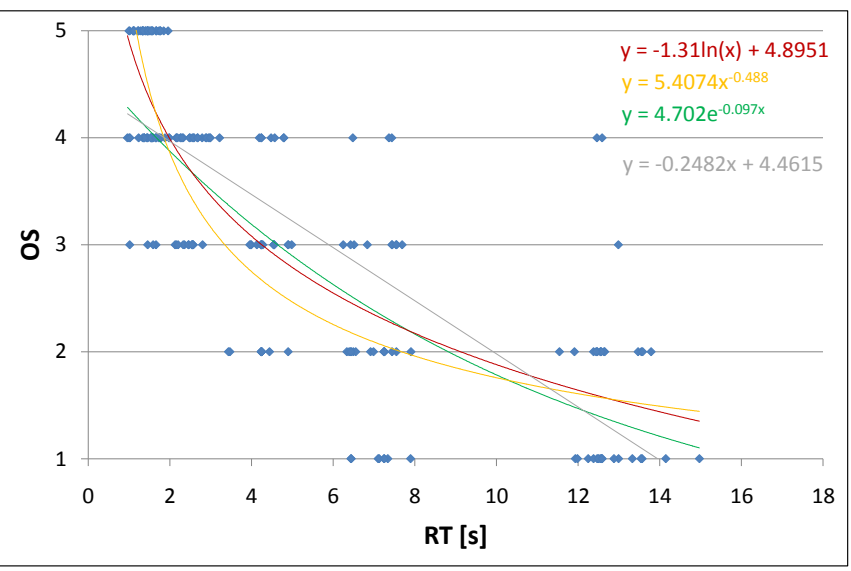

Fig. 4. OS for corresponding RT for confirm test with exponential trendline.

Oslo. The BWs used were maximal BW (which changes with the used type of network connection), 2000, 1000, 750, 500, 350 , and $250 \mathrm{~B} / \mathrm{s}$. Bandwidths under $250 \mathrm{~B} / \mathrm{s}$ gave a timeout and were not usable in the experiment. In this experiment we also used different non-fixed line network connections, such as wireless and mobile, together with the traffic shaper in order to force a greater variety of RTs. However, the RTs did not go much below $4 \mathrm{~s}$ because of the wireless or mobile network connection.

The tests were performed on the login procedure on the OpenID server web page. However, the users were shown how it should work when accessing, or using, it from another web page, such as MyGeolog.com, like in the first experiment. This was done to ensure that the user knew what he or she was doing and what the purpose of the task was. This time all of the authentication procedure was ranked, i.e. the login at the server web page. The user was asked to give a ranking, based on the RT of that particular task, between 1 and 5, for their experience of the security.

The RTs were recorded in Firefox in a similar fashion as in the browsing test setup. In this experiment the addon Firebug Net Panel History Overlay [16] for the add-on Firebug [17] (the older version 1.2.0b6, which supports the history add-on) was used for recording the RT for web pages. Firebug gives detailed information on all times of a web page loading. Though, the authentication was done via a Java applet on the OpenID server web page before the actual web page started loading, and the times for java applet communication is not recorded by neither Fasterfox nor Firebug. This was then solved in the supplicant (see Fig. 2) with code that, in a log file, wrote detailed timestamps at the client side for all communication steps of the authentication.

Both the timestamps in Firebug and the timestamps in the $\log$ file were taken from the client computer, so the timestamps could then be compared to make sure that they would give the correct total RT. The total RT for the login procedure, as the user perceived it, was then the time for the authentication added to the time for loading the web page.



Fig. 5. OS for corresponding RT for login test with exponential trendline.

\section{RESUlts}

The user experiments gave a couple of noteworthy results, both qualitative and quantitative, that are presented in this section. For the quantitative results of the login experiment we provide an equation for producing a MOS from RTs in future experiments with authentication solutions. This equation comes from the trendline for the MOS from the user experiments performed in this study. Out of the candidates for the trendline, an exponential function had the bests fit and was therefore chosen according to the results in [5], and [3]. The trendlines were matched to the (OS, RT) pairs according to the least square principle, and is shown together with the coefficient of determination $R^{2}$ as indicator: the closer $\left|R^{2}\right|$ gets to one, the better the trend is captured.

\section{A. Quantitative results}

The experiments gave OSs from all users that were then mapped to the corresponding RTs. These are shown, for the confirm experiment in Fig. 4, and for the login experiment in Fig. 5 together with matchings, or trendlines, of the values. The same matchings as in [2] are used, derived from regressions of $y$ vs. $x$ (linear), $y$ vs. $\ln (x)$ (logarithmic), $\ln (y)$ vs. $x$ (exponential), and $\ln (y)$ vs. $\ln (x)$ (power).

In the web test [2], were users were waiting for pictures to download completely, the exponential and logarithmic matchings are of similar quality and outperform both the linear and the power matching.

In the confirm test, the logarithmic matching matches best, followed by power, exponential and linear matching. Fig. 4 reveals that both logarithmic and power matchings better approximate the behaviour for small RTs. Indeed, all nonlinear matchings take into account the convex trend of the measurements, implying a slower decay as RT values increase (i.e. $y^{\prime}(x)<0$, but $y^{\prime \prime}(x)>0$ ).

In the login test, the exponential matching is superior to power, logarithmic and linear matching. In Fig. 5 it is shown that the non-linear matchings are found pretty close to each other. In particular, the comparison of the exponential matchings for confirm and login test reveals similar sets of 
TABLE II

TRENDLINE EQUATION WITH RESPECTIVE COEFFICIENT OF DETERMINATION $\left(R^{2}\right)$.

\begin{tabular}{|c||c|c|c|}
\hline \multicolumn{1}{|c||}{} & Type & $R^{2}$ & Regression \\
\hline \hline \multirow{4}{*}{ Web test } & Exponential & 0.990 & $y=4.836 \mathrm{e}^{-0.150 x}$ \\
\cline { 2 - 4 } & Logarithmic & 0.988 & $y=-1.426 \ln (x)+4.469$ \\
\cline { 2 - 4 } & Power & 0.912 & $y=5.339 x^{-0.638}$ \\
\cline { 2 - 4 } & Linear & 0.966 & $y=-0.318 x+4.158$ \\
\hline \hline Confirm test & Exponential & 0.618 & $y=4.702 \mathrm{e}^{-0.097 x}$ \\
\cline { 2 - 4 } & Logarithmic & 0.691 & $y=-1.31 \ln (x)+4.895$ \\
\cline { 2 - 4 } & Power & 0.643 & $y=5.407 x^{-0.488}$ \\
\cline { 2 - 4 } & Linear & 0.966 & $y=-0.2482 x+4.462$ \\
\hline \hline \multirow{2}{*}{ Log in test } & Exponential & 0.807 & $y=4.836 \mathrm{e}^{-0.107 x}$ \\
\cline { 2 - 4 } & Logarithmic & 0.720 & $y=-1.687 \ln (x)+5.576$ \\
\cline { 2 - 4 } & Power & 0.791 & $y=11.065 x^{-0.860}$ \\
\cline { 2 - 4 } & Linear & 0.705 & $y=-0.206 x+3.921$ \\
\hline
\end{tabular}

coefficients in Table II, i.e 4.702 versus 4.836 as factor, and -0.097 versus -0.105 in the argument of the exponential function. This motivates us to focus on the exponential matching in the sequel.

Comparing the confirm and login tests with regular web usage [2], a certain difference in the argument of the exponential function can be seen. In the web case, the curve is decaying more quickly $(-0.15$ instead of -0.1$)$. As seen in Fig. 4, some users do not really think that the service is bad despite the high RT, and still at up to 12 or $13 \mathrm{~s}$ they think it is fair (3) or even good (4). This points at slightly increased user patience in the case of logins as compared to web usage. A similar evidence is seen from the comparison of the power matchings between web test and confirm test in Table II, where the latter displays a slower decay (power -0.488 instead of -0.638 ).

\section{B. Qualitative results}

Users in the login tests were asked what they thought of the service of OpenID. The results were, of course, a bit different between the users, but surprisingly enough many of the users were united in some points. These are presented below and then briefly discussed.

- "I would not use it in the beginning."

- "Is it really safer than passwords? I can lose the SIMcard."

- "Good solution IF it works!"

- "If I forget my phone at home I wont be able to log in anywhere!"

- "If I don't pay for the service I can wait an extra second."

- "When it is too fast, or too slow, it feels like something is wrong."

Uncertainty of what the service does and what it means for the users, how it really works, could be solved with detailed information. The risk of losing the SIM-card, which in some cases are a reality could be compared to losing a "regular" SIM-card in a cellular phone. The last point is perhaps the most interesting. If a user chooses to pay for a service, the expectations are probably high, but if the service is free the



Fig. 6. User perception profile: Frequency of OSs from RTs for different BWs.

user might be more patient when experiencing for example higher RTs.

\section{User perception profile}

The approximation formula $O S=f(R T)$ is used in order to construct a direct link between performance of the authentication solution under study and the (predicted) user perception. The exponential approximation is used as it has shown to be the most generic of all the matchings, in particular with respect to the obtained parameters.

Fig. 6 shows, for all different test cases from $250 \mathrm{kbps}$ to $2 \mathrm{Mbps}$ of bandwidth, what the predicted OS would be with our user perception profile, or user model, as tool. The system in question, which is the same system as in the previous experiments, was tested with different bandwidth constraints. The RTs that was logged for the different bandwidths were then transformed into OSs according to the formula $O S=4.7 \mathrm{e}^{-0.1 R T}$, as shown in Fig. 6. It can be seen that the case of $250 \mathrm{kbps}$ would not be accepted by the users and that bandwidths from $700 \mathrm{kbps}$ and higher would be well accepted by users. So, with the user model it is possible to benchmark systems, within the QoE domain, before they are put in use.

\section{DISCUSSION}

When comparing the exponential trendlines in the case of pure web experience, pure login experience and the case with experience of the last part of a login, we saw that the equations were similar, but a bit higher for both of the login experiments (see Table II). This would imply that users doing a login are a bit more patient than users of regular web pages, which also applies for other waiting times in the context of IT services [18].

The results imply a direct relationship between the performance of the authentication method and the corresponding user ranking, which enables the expression of performance directly in expected user ranking of login, i.e. QoS can be directly matched to QoE. This kind of models for expressing relationships between QoS and QoE are valuable for companies, such as different kinds of service providers, on the 
purpose of QoE benchmark testing systems (as shown in Fig. 6) before delivery or release.

However, models for producing QoE from QoS or QoD are also hard to produce because of the large number of parameters that must be taken into consideration. Parameters that should be considered in such a model are not only RTs and user rankings, but for example a set of parameters that affect the user expectations, like network connection and different kinds of user background.

Another very interesting aspect of Fig. 4 and 5 are the point when the ratings that are lower than 3 (fair) start appearing. A company providing a service would not want to get rankings of 2 (poor) and definitely not rankings of 1 (bad). From both graphs (Fig. 4 and 5) we can clearly see that around 4 seconds users already start giving the grade 2 . In the graph from the confirm test (Fig. 4) we can also see that there are rankings of 1 at about 7 seconds, and rankings of 2 already at about 4 seconds. The latter implies that service providers should be careful already when RTs start growing larger than 4 seconds.

\section{CONCLUSION AND FUTURE WORK}

This paper shows a study of user experience of web pages including the security feature of authentication, or as it is called by users: login. After several experiments, both on web experience with and without login, we came to the conclusion that there are differences on what users think about delay in web experience with security as compared to web experience without security. The results that we got from the experiments follow the line of the previous studies regarding QoE of web browsing, such as [3] and also other similar studies, such as [4] and [5], however, with different time constants. The results indicate slightly higher user patience when security is involved, as in the login experiment presented in this paper.

The best fitting trend of the QoE, for the login and confirm experiments, follow an exponential shape that was presented as a user model. Thus, delay in a system with a web page as user interface can easily be expressed as QoE by using this model. The question of whether a user will be satisfied with a certain service can be answered without extensive user experiments, which will save a lot of time, thus money.

Within the larger study, that will follow the work presented in this article, a user experience model will be used to further evaluate different authentication methods, based on their RTs. A user will not know what authentication method is used and the experience of the user will be based on the RTs. As long as the user interface and the environment is the same as in the first experiments, the model that was produced from the first experiments can still be used. The user interface used in the first experiments was a simple login on a web page with no specific requirements to environments, which leaves us with a large area of use for the user model. The latter, in turn, will be refined if necessary, as new pairs of OS and RT measurement will become available.

The next step is the evaluation of different authentication methods together with OpenID. The user model presented in this paper will be used on the performance parameters of the evaluation to receive a QoE evaluation result. Though, e.g. for the One-time password (OTP) authentication method, new user experiments still need to be conducted, since that would mean a totally new experience for the user, in the context of the OpenID. A OTP authentication will include e.g. a cell phone, on which the OTP will be received.

\section{ACKNOWLEDGMENT}

The authors would like to thank Syed Usman Ali for his contribution and hard work on carrying out the first set of experiments in his Master Thesis work.

\section{REFERENCES}

[1] OpenID Foundation website: http://openid.net [Online] [Cited on 201004-02]

[2] ITU-T Recommendation P.10/G.100 (2006)/Amendment 2 (07/2008). "Vocabulary for performance and quality of service. Amendment 2: New definitions for inclusion in Recommendation P.10/G.100".

[3] J. Shaikh, M. Fiedler and D. Collange: Quality of Experience from user and network perspectives. In Annals of Telecommunications "Quality of Experience - 1 Metrics and performance evaluation", vol 65, issue 1-2, January/ February 2010.

[4] S. Eriksén, C. Eliasson, M. Fiedler, S. Chevul and A. Ekelin: Mapping service quality comparing quality of experience and quality of service for Internet-based map services. In Proceedings of the 30th Information Systems Research Seminar in Scandinavia (IRIS 2007), August 2007, Tampere, Finland.

[5] T. Hoßfeld, M.Fiedler and P. Tran-Gia. "A Generic Quantitative Relationship between Quality of Experience and Quality of Service". IEEE Network Special Issue on Improving QoE for Network Service, March 2010.

[6] C. Eliasson, M. Fiedler and I. Jørstad: A criteria-based evaluation framework for authentication schemes in IMS. In proceedings of the 4th International Conference on Availability, Reliability and Security (AReS 2009), March 2009, Fukuoka, Japan, pp. 865-869.

[7] M. Fiedler, ed. EuroNGI Deliverable D.WP.JRA.6.1.1: State-of-the art with regards to user-perceived Quality of Service and quality feedback. May 2004.

[8] P. Reichl: From 'Quality-of-Service' and 'Quality-of-Design' to 'Qualityof-Experience': A Holistic View on Future Interactive Telecommunication Services. In Software, Telecommunications and Computer Networks, 2007.

[9] ITU-T Recommendation P.862 (2001). "Perceptual evaluation of speech quality (PESQ): An objective method for end-to-end speech quality assessment of narrow-band telephone networks and speech codecs".

[10] ITU-T Recommendation G.107 (2000). "The E-model, a computational model for use in transmission planning".

[11] G. Rubino: "Quantifying the Quality of Audio and Video Transmissions over the Internet: the PSQA Approach". In J. Barria (ed.), Design and Operations of Communication Networks, Imperial College Press, 2005.

[12] ITU-T Recommendation P.800 (1996). "Methods for subjective determination of transmission quality".

[13] M. Fiedler, ed. EuroNGI Deliverable D.WP.JRA.6.1.1: State-of-the art with regards to user-perceived Quality of Service and quality feedback. May 2004. http://www.eurongi.org [Online] [Cited on 2010-05-31]

[14] M. Fiedler, C. Eliasson, S. Chevul, and S. Eriksén. Quality of Experience and Quality of Service in a Service Supply Chain. EuroFGI IA.7.6 Workshop on Socio-Economic Issues of Future Generation Internet, Santander, Spain, June 2007.

[15] Add-ons for Firefox. Fasterfox 2.0.0 by Tony Gentilcore: https://addons.mozilla.org/en-US/firefox/addon/1269 [Online] [Cited on 2010-04-02]

[16] Add-ons for Firefox. Firebug Net Panel History Overlay 0.4 .0 by Mihailo Lalevic: https://addons.mozilla.org/en-US/firefox/addon/7189/ [Online] [Cited on 2010-04-02]

[17] Add-ons for Firefox. Firebug Version History (Version 1.2.0b6) by Joe Hewitt, et al.: https://addons.mozilla.org/enUS/firefox/addons/versions/1843\#version-1.2.0b6 [Online] [Cited on 2010-04-02]

[18] J. Nielsen. "Usability Engineering". Morgan Kaufman, San Francisco, 1994. 